\title{
A Framework for Lightweight QoS Provisioning: Network Planes and Parallel Internets
}

\author{
N. Wang ${ }^{1}$, D. Griffin 2 , J. Spencer ${ }^{2}$, J. Griem ${ }^{2}$, J. Rodríguez Sánchez ${ }^{3}$, M. Boucadair ${ }^{4}$, E. Mykoniati ${ }^{5}$, B. Quoitin ${ }^{6}$, \\ M. Howarth ${ }^{1}$, G. Pavlou ${ }^{1}$, A. J. Elizondo ${ }^{3}$, M. L. García Osma ${ }^{3}$ and P. Georgatsos ${ }^{5}$ \\ ${ }^{1}$ University of Surrey, United Kingdom \\ ${ }^{2}$ University College London, United Kingdom \\ ${ }^{3}$ Telefónica Investigación y Desarrollo, Spain \\ ${ }^{4}$ France Telecom R\&D, France \\ ${ }^{5}$ Algonet S. A. Greece \\ ${ }^{6}$ Université catholique de Louvain, Belgium
}

\begin{abstract}
In this paper we introduce the concepts of Network Planes and Parallel Internets, with the objective of designing and implementing a lightweight solution for viable end-to-end QoS provisioning. The proposed solution can be deployed with very small incremental additions to the existing best-effort Internet. Through Network Plane engineering and interconnection, mainly by means of intra- and inter-domain routing differentiation, end-to-end service differentiation across the Internet can be achieved.
\end{abstract}

\section{INTRODUCTION}

The Internet is evolving to become a multi-service platform that is able to support diverse multimedia applications. In order to better support emerging services such as video streaming, IP Telephony, Virtual Private Networks (VPNs) etc., Quality of Service (QoS) awareness has become an essential requirement for guaranteeing end-to-end performance. Differentiated Services (DiffServ) [1] has been regarded as a promising attempt based on differentiated forwarding mechanisms that can be used to build a small number of QoS classes. Although DiffServ can serve as a practical control/data plane forwarding mechanism that supports QoS differentiation, a comprehensive solution for QoS provisioning is still missing due to the lack of other supporting techniques such as QoS-aware routing, resource management and also appropriate cooperation between individual administrative domains for enabling end-to-end QoS across the Internet.

The recent emergence of new players, such as Skype and Yahoo! indicates the separation of service and network plane administrations, leading to a distinction between the Service Provider (SP) and IP Network Provider (INP) business roles. By decoupling these roles, the market for added-value IP-based services will open up and new multimedia services will become widely available, in addition to the plain Internet access services offered today. A key aspect in this multi-provider environment is the vertical cooperation of SPs with INPs and the horizontal cooperation between INPs and between SPs for inter-provider service delivery. Although added-value services can be provided in a single administrative domain with appropriate network and traffic engineering techniques (e.g., through MPLS), lightweight approaches are still desired for more scalable QoS provisioning solutions. Moreover, interconnection agreements and cooperative network engineering are needed in addition for end-to-end service provisioning across multiple administrative domains, which has not yet been fully investigated till now.

The EU IST AGAVE project [3] aims to design and implement a lightweight approach for achieving end-to-end QoS across the Internet. In this paper, we describe the AGAVE QoS provisioning approach, which assumes the decoupling of SP and INP business roles. Specifically, the SP offers IP-based services to end customers relying on INPs for fulfilling the IP connectivity aspects of the offered services, while the INP provides IP connectivity to SPs and not directly to end customers. We propose the concept of Parallel Internets (PIs) as an innovative way to enable end-to-end service differentiation at the IP level in terms of not only traditional QoS such as delay and loss, but also resilience and availability. Specifically, Parallel Internets are coexisting parallel networks composed of interconnected per-domain planes. We call the distinct planes within each domain Network Planes (NPs). Network Planes are setup to transport traffic flows from services with common connectivity requirements. The traffic delivered within each Network Plane has particular treatment in both forwarding and routing so that service differentiation across NPs is enabled in terms of edge-to-edge QoS, availability and resilience.

\section{A LIGHTWEIGHT FRAMEWORK FOR QOS PROVISIONING}

We introduce the concept of Network Planes to denote and to differentiate the IP transfer treatment experienced by IP flows when crossing an IP realm managed by a single INP. NPs are internal to INPs and they can be created proactively or to support the specific requirements imposed by a particular SP. An NP can be used to convey traffic from one or several services managed by the same or different SPs. The INP and the SP agree on the classification, invocation and access rules for the traffic associated with the service managed by the SP but the association between 
service requirements and $\mathrm{NP}$ is an internal $\mathrm{NP}$ matter, not visible to SPs.

A Network Plane is defined as the combined tuning of several processes, across one or more of the dimensions listed below:

(1) The Routing dimension. The treatment of the IP packets can be differentiated on the basis of routing policies and configuration. Differentiated routing can be implemented at several levels:

- Dedicated topology: Dedicated topology can be physical or logical topology. Therefore, several routing adjacencies can be maintained. These adjacencies are, for instance, the result of including or excluding certain nodes or links;

- Dedicated routing selection process: Several route selection processes can be configured, each of them being dedicated to one or multiple NPs. These multiple route selection processes can operate either on the same topology or on dedicated topologies. The behaviour of each route selection process is different;

- Different fast reroute procedures: This is specifically for resilience purposes. The routing process corresponding to a topology can be enhanced to support fast rerouting when failures occur at the given topology;

- Different policies and metrics: An alternative to implement differentiated routing is to have a dedicated metric value for each NP. Therefore, the selected path will not be the same towards the same destination for the traffic of different NPs.

(2) Forwarding dimension. At the forwarding level, an INP can engineer its IP forwarding mechanisms so as to have distinct forwarding behaviours by configuring different strict priority or fair-queuing classes over a common scheduler, assigning dedicated scheduling resources, differentiating dropping policies, differentiating failure detection means, etc. A typical example would be the DiffServ paradigm.

(3) Resource Management dimension. The treatment experienced by IP packets can be differentiated by (a) having different shaping and policing rules, and (b) the amount of admitted traffic given the capacity, also denoted as overprovisioning factor.

The concept of Parallel Internets is introduced as an innovative way to enable end-to-end service differentiation in terms of network QoS, resilience and availability. Specifically, Parallel Internets are coexisting parallel networks composed of interconnected Per-Domain Network Planes. Parallel Internets are constructed from the perspectives of each INP, by configuring for each Network Plane different inter-domain routes to certain destinations, based on local criteria. For each Network Plane, traffic may exit the INP domain through a different AS Border Router (ASBR), or through different portions of the same interdomain link (e.g. based on forwarding differentiation). Moreover, different Network Planes may use different intra-domain routes. As a result, traffic classified at ingress nodes to different Network Planes may be delivered through dedicated intra- and interdomain routes. This way, INPs would be able to support different levels of availability, resilience and QoS to remote destinations by different inter-domain routes appropriate to the requirements of the particular NP and the service traffic carried over it.

\section{NETWORK PlaNE IMPLEMENT ATION}

\section{A. Network Plane Engineering}

The engineering of Network Planes is achieved through tuning one or multiple dimensions mentioned above. In this section, we provide a set of techniques, specifically in the routing dimension, that may be used alone or combined with other ones (like DiffServ-based forwarding). It is worth mentioning that, these routing techniques have different capabilities in providing QoS, availability and resilience guarantees, and INPs may select the most appropriate routing mechanism(s) to implement a specific Network Plane according to service requirements.

\section{1) Multi-topology based Traffic Engineering}

Multi-Topology routing in OSPF (MT-OSPF) [4] allows a router to belong to multiple topologies, identified by a Multi-Topology Identifier (MT-ID). Each link is assigned a dedicated link weight for each topology it belongs to, and, therefore, traffic assigned to different topologies can be routed independently over the same physical INP infrastructure. MT-OSPF is an ideal mechanism to implement multiple routing planes and the differentiated routing aspect of NPs. When combined with differentiated forwarding it is possible for different topologies to deliver different QoS levels at the same time as optimising the network through load balancing or meeting other goals of NPs such as providing multiple levels of resilience to failures.

While MT-OSPF identifies the mechanisms for deploying NPS in the routers, NPs need to be designed by an off-line traffic engineering and resource management process that can ensure the network is optimised to meet the traffic demands. As part of this process routers and links need to be allocated to NPs and a dedicated set of MT-OSPF link weights needs to be assigned for each routing plane.

Apart from the offline NP engineering paradigms, online routing for dynamic load balancing and fast rerouting in case of link congestions or failures can be also achieved through multitopology routing within one NP or even vertically across multiple NPs. A typical example is to maintain dedicated MT-OSPF routing topologies within one specific NP in order to enable edgeto-edge path diversity. Once the NP is invoked, traffic assigned to this NP can be dynamically tuned across compatible MT-OSPF routing topologies according to the monitored QoS/TE performance of this Network Plane.

\section{2) Multi-path Routing with Dynamic Variance (MRDV)}

Multipath Routing with Dynamic Variance (MRDV) $[5,6]$ introduces multipath dynamic routing to current distributed IGP routing processes. This algorithm uses a variable number of alternative routes towards a destination, where the number of paths increases with measured load. Thus, traffic will be distributed among several paths, reducing congestion and leading to a better use of network resources. Alternative paths are considered to be suitable when their metric is sufficiently similar to the shortest path as defined by:

$$
M \leq M_{\min } \cdot V
$$

where $M$ is the metric of the path, $M_{\min }$ is the metric of the optimal path, and $V$ is the variance parameter. 
MRDV adjusts the variance parameter dynamically, according to the average load that the router detects in the next hop of the optimal path towards the destination. As the variance parameter value increases new paths are introduced and load is distributed among the suitable paths in inverse proportion to the path cost: the less cost a path has, the more traffic it receives.

In order to implement NPs to cater for traffic with different QoS requirements, a separate variance parameter is maintained for each traffic class. When a router calculates a variance parameter value for a particular traffic class, it considers the load generated by that traffic class and that offered by all the higher priority traffic classes. Therefore, the traffic of lower priority classes will be distributed over more paths and higher priority classes will have more traffic on paths with lower cost. The overall amount of traffic class differentiation introduced is configured by adjusting the corresponding parameters this algorithm introduces $[5,6]$.

This mechanism is decentralized with routers directly measuring the load on their links. This facilitates deployment by retaining compatibility with current intra-domain routing protocols, therefore allowing a scalable and gradual deployment. However, distributed multi-path techniques, where different paths with different costs are considered, potentially introduce routing loops. Therefore, a protocol to delete loops has been included in MRDV: the LAP (Loop Avoidance Protocol) is studied thoroughly in [5].

\section{B. Creating Parallel Internets}

Since earlier stages of IP networking, several proposals have aimed to capture service requirements on routing, especially interdomain routing, and to specify solutions meeting these requirements. In 1996, the Nimrud initiative was launched within the IETF with the ambition to provide service-specific routing in the presence of multiple constraints imposed by service providers and users. RFC1992 [10], one of the key documents produced by the Nimrud working group, states that inter-network connectivity and services should be represented in the form of maps at multiple level of abstraction. Unfortunately, we didn't see complete proposals to implement this mandate on operational networks. Our solution introduces the concept of Network Planes which are one step forward to implement service differentiation within the domain of a single INP. However, in order to expand the service beyond the boundaries of a single INP, additional means should be adopted so as to ensure coherence and consistency of treatment when crossing several INP domains such as meta-QoS-classes [11] and QoS-enhanced BGP [8]. The use of QoS-enhanced BGP, optionally with the concept of meta-QoS-classes is able to offer either statistical or loose end-to-end $\mathrm{QoS}$ guarantees across multiple INPs. In addition, we also introduce inter-domain IP tunnelling mechanisms for providing better-than-best-effort services (i.e. enhanced traffic performance without any guarantees).

\section{1) Meta QoSClasses}

The philosophy behind the meta-QoS-class concept relies on a universal and common understanding of QoS-sensitive applications' requirements. Wherever end-users are connected, they experience the same QoS difficulties and are likely to express very similar QoS requirements to their respective providers. Globally confronted with the same customers' requirements, providers are likely to design and operate similar Network Planes, each of them being particularly designed to support services with the same constraints, especially QoS constraints, resilience and traffic protection means.

INPs use the meta-QoS-class concept to map and bind their NPs to external ones. An INP goes through several steps to expand its internal NPs. First, it classifies its own NPs based on meta-QoSclasses. Then, it learns about available meta-QoS-classes advertised by its neighbours. Third, it establishes an agreement with its neighbour to be able to send traffic that will be handled according to the agreed meta-QoS-classes. An NP can be bound only with a neighbour NP that is classified as belonging to the same meta-QoS-class.

A hierarchy of meta-QoS-classes can be defined for a given type of service (e.g. IP Telephony with different quality levels). A given NP can be suitable for several meta-QoS-classes. In this case, several code points (e.g., DSCPs in DiffServ ${ }^{1}$ ) are likely to be associated with the same NP in order to differentiate between traffic classes. Several NPs in a given INP domain can be classified as belonging to the same meta-QoS-class.

\section{2) $Q o S$-enhanced $B G P$}

QoS-enhanced BGP is an enriched version of the BGP protocol. It has been proposed to support QoS requirements for the deployment of QoS-based services across several domains managed by distinct INPs. QoS-Enhanced BGP is used to convey routing and QoS information between the domains of directly interconnected INPs. Two modes of activation of QoS-enhanced BGP are valid so as to extend the Network Planes beyond the boundaries of a single provider. The first alternative consists of configuring several QoS-enhanced BGP sessions, each dedicated to a given Network Plane. And the second alternative which consists in activating a single QoS-enhanced BGP session which will multiplex reachability information of all involved Network Planes.

In order to exchange QoS performance characteristics, QoSenhanced BGP uses the following messages as defined in [8]:

1. QoS service capabilities: since peering entities need to know about each other's QoS service capabilities, QoS-enhanced BGP allows to negotiate the capabilities that a peer domain provides, and indicates what information can potentially be carried by the QoS-enhanced BGP messages;

2. QoS Class identifier: this is used to distinguish the Network Planes that have been bought by/from service peers.

3. QoS performance characteristics: these are a set of QoS characteristics values, such as one-way packet loss and delay and inter-packet delay variation. QoS-enhanced BGP supports a set of QoS performance characteristics to be sent in one single QoS-enhanced BGP UPDATE message.

\section{3) IP tunnelling}

A common method used by ASes to engineer the flow of their inter-domain traffic is to establish peerings with other ASes [12]. For common practice, those peerings are established either

\footnotetext{
${ }^{1}$ It should be noted that DSCPs are only used for identifying traffic classes but the traditional DiffServ forwarding mechanisms are not necessarily compulsory.
} 
through direct private links between the two ASes or over an interconnection point. An eBGP session is used over the peering link to advertise the prefixes that are reachable via each AS. In addition to this, BGP peerings are established manually by changing the routers configurations on both ends by hand. However, manual operations are error-prone and slow. In addition, the time of establishment of a new peering is often on the order of magnitude of several days or weeks.

In our framework, we consider the extension of such peering mechanisms to non-adjacent ASes, through the utilization of Virtual Peerings [7]. A Virtual Peering is a peering built on dynamically established uni-directional IP tunnels between two cooperating, but non-adjacent, ASes. These IP tunnels are used by the source AS to send packets to the destination AS via chosen ingress routers in the destination AS. The only requirement to be able to deploy such IP tunnels is that the remote ingress routers IP addresses be routable separately. Today, an increasing number of ASes already establish peerings with non-adjacent ASes by relying on L2VPNs (see [13], for instance). Emulating such pointto-point links using tunnels is currently investigated by the IETF in the PWE3 working group [14].

In AGAVE, we investigate the utilization of Virtual Peerings as a means to better engineer the inter-domain traffic of ASes. We envision several applications of Virtual Peerings. A first example would be to use Virtual Peerings to balance the load of traffic received by an AS over its access links. Another example would be to forward traffic towards a remote destination along a path which has a better quality than the default BGP-learned routes. A typical use case would be to engineer a lower latency path between two SIP proxies. Using Virtual Peerings in this way would allow the provision of better than best-effort services without the need for end-to-end signalling and reservation as proposed with MPLS/RSVP-TE solutions.

The advantage of using IP tunnels for inter-domain traffic engineering is twofold. First, IP tunnels allow to leverage the Internet path diversity. With BGP, only a small subset of the available paths is learned by the ASes, due to the routing policies enforced by the intermediate ASes but also to the path-vector nature of BGP. Indeed, BGP routers currently only allow a single best route to be propagated to their neighbours for each destination. Second, the cooperation of intermediate ASes is not required to deploy IP tunnels. The forwarding decisions are taken by the cooperating ASes at the endpoints of the tunnel only. For this reason, IP tunnels can readily be deployed without the need for the whole Internet infrastructure to be updated.

\section{SUMMARY}

In this paper, we have introduced the concepts of Network Planes and Parallel Internets for the purposes of lightweight QoS provisioning across the current best-effort Internet. The proposed framework assumes the decoupling of Service Providers (SPs), who offer IP-based services, from IP Network Providers (INPs) who own the actual IP network resources. From the viewpoint of the INPs, in order to satisfy heterogeneous QoS requirements demanded by SPs, a set of Network Plane Engineering solutions are designed and implemented, namely QoS aware multi-topology
IP traffic engineering and Multi-path Routing with Dynamic Variance (MRDV). In addition, approaches for horizontally binding individual Network Planes across multiple autonomous domains for creating QoS aware Parallel Internets have also been described for enabling end-to-end QoS differentiation. Specifically, the concept of meta-QoS class and the QoSenhanced BGP protocol are described for inter-domain QoS provisioning. The IP tunnelling approach is also introduced for providing better-than-best-effort services through virtual peering between remote $\mathrm{NPP}$.

\section{REFERENCES}

[1] S. Blake et al, "An Architecture for Differentiated Services", RFC 2475

[2] Y. Rekhter and T. Li, "A Border Gateway Protocol 4 (BGP-4)", IETF RFC 1771, March 1995.

[3] M. Boucadair et al, "Parallel Internets Framework", AGAVE Deliverable D1.1, September 2006

[4] P. Psenak, A. Roy, L. Nguyen, P. Pillay-Asnault, "MT-OSPF: Multi Topology (MT) Routing in OSPF", Internet draft, draft-ietf-ospf-mt06.txt, February 2006

[5] Ramón-Salguero, F.J., J. Enríquez-Gabeiras, J. Andrés-Colás, A. Molíns-Jiménez: "Multipath Routing with Dynamic Variance", COST 279 Technical Report TD02043 (2002).

[6] Callejo-Rodríguez, M.A., J. Andrés-Colás, G. García-de-Blas, F.J. Ramón-Salguero, J. Enríquez-Gabeiras: "A Decentralized Traffic Management Approach for Ambient Networks Environments", 16th IFIP/IEEE International Workshop on Distributed Systems: Operations and Management (DSOM) 2005, 145-156. Springer.

[7] B. Quoitin and O. Bonaventure, "A cooperative approach to interdomain traffic engineering", Proc. $1^{\text {st }}$ Conference on Next Generation Internet Networks Traffic Engineering (NGI) 2005

[8] M. Boucadair, "QoS-Enhanced Border Gateway Protocol", InternetDraft, draft-boucadair-qos-bgp-spec-01.txt, July 2005

[9] Fortz, B., and M. Thorup, "Internet Traffic Engineering by Optimizing OSPF Weights", Proc. IEEE INFOCOM 2000

[10] I. Castineyra, N. Chiappa, and M. Steenstrup, "The Nimrod Routing Architecture", Internet Engineering Task Force, RFC1992, 1996

[11] P. Levis and M. Boucadair, "The Meta-QoS-Class concept", InternetDraft, draft-levis-meta-qos-class-00.txt, June 2005

[12] Bartholomew S., "The Art of Peering", 2000

[13] LINX, "Pseudowire / Layer-2 Connect", https://www.linx.net/www_public/our_services/layer2_connect/?sear chterm=martini, 2006

[14] Bryant S. and Pate P., "Pseudo Wire Emulation Edeg-to-Edge (PWE3) Architecture", RFC3985, 2005 